EPJ Web of Conferences 80, 00014 (2014)

DOI: $10.1051 /$ epjconf/20148000014

(C) Owned by the authors, published by EDP Sciences, 2014

\title{
Hybrid exotic mesons in soft-wall AdS/QCD
}

\author{
Loredana Bellantuono ${ }^{1,2, a}$ \\ ${ }^{1}$ INFN-Sezione di Bari, via Orabona 4, 70126 Bari, Italy \\ ${ }^{2}$ Dipartimento di Fisica, Università degli Studi di Bari Aldo Moro, via Orabona 4, 70126 Bari, Italy
}

\begin{abstract}
Hybrid mesons with exotic quantum numbers $J^{P C}=1^{-+}$are examined in softwall AdS/QCD. The predicted mass spectrum is compared to the measured values of the candidates $\pi_{1}(1400), \pi_{1}(1600)$ and $\pi_{1}(2015)$. Thermal effects are analysed through the spectral function in the AdS-Black Hole model, and the differences with the HawkingPage description are discussed.
\end{abstract}

\section{Introduction}

Quantum Chromodynamics (QCD) describes strong interactions among quarks as processes in which colored gluons are exchanged. By virtue of their own color charge, gluons also strongly interact with quarks and among themselves. For this reason, a meson should be considered as a linear superposition of color-singlet bound states $\left|q \bar{q}^{\prime}\right\rangle,\left|q q \bar{q}^{\prime} \bar{q}^{\prime}\right\rangle,\left|q G \bar{q}^{\prime}\right\rangle,|G G\rangle, \ldots$, comprising quarks ( $q$ ), antiquarks $(\bar{q})$ and gluons $(G)$ as constituents [1]. These states can be classified, respectively, as quark model "quarkonia", "multiquarks", "hybrids", "glueballs", and so forth. These structures determine angular momentum, parity and charge-conjugation quantum numbers $J^{P C}$ of the meson, and they yield also exotic combinations not included in the quark model $q \bar{q}^{\prime}$ picture.

Hybrid configuration, composed by a quark-antiquark pair plus a constituent gluon, accounts for either ordinary or exotic $J^{P C}$ quantum numbers. Therefore, experimental evidence of these states can come from overpopulations of the ordinary $J^{P C}$ spectra compared to quark model prediction, or from the detection of exotic states. The first strategy seems unfruitful, because of the densely populated spectrum of light mesons in the mass region between 1 and $2 \mathrm{GeV}$, and the broad nature of the states involved [2]. On the other hand, states that can have exotic quantum numbers are multiquarks and hybrids. They have been searched in experiments aiming at the detection of their decay products, but the analysis has revealed cumbersome. The identification of exotic resonances would be a strong argument supporting the existence of hybrid bound states. Several QCD models identify the meson with $J^{P C}=1^{-+}$as the lowest-lying exotic state, with mass predictions varying between 1.5 and 2.2 $\mathrm{GeV}$ [2]. Currently, in the light quark sector there are three candidates for hybrid $1^{-+}$states: $\pi_{1}(1400)$, $\pi_{1}(1600)$ and $\pi_{1}(2015)$. Their measured masses are $M\left(\pi_{1}(1400)\right)=1354 \pm 25 \mathrm{MeV}, M\left(\pi_{1}(1600)\right)=$ $1662_{-9}^{+8} \mathrm{MeV}$ and $M\left(\pi_{1}(2015)\right)=2014 \pm 20 \pm 16,2001 \pm 30 \pm 92 \mathrm{MeV}$ [3]. Further information on the detection of such states can be found in the bibliography of [4]. The nature of $1^{-+}$candidates is still a debated issue, and new experiments with higher statistics and better acceptance are expected to improve present understandings [2].

\footnotetext{
ae-mail: loredana.bellantuono@ba.infn.it
} 
I examine the $J^{P C}=1^{-+}$mesons in the holographic approach, a framework inspired by the AdS/CFT correspondence. This conjecture relates type IIB string theory in an $A d S_{5} \times S^{5}$ space $\left(A d S_{5}\right.$ is a 5-dimensional anti-de Sitter space and $S^{5}$ is a 5-dimensional sphere) with $\mathcal{N}=4$ SuperYang Mills theory in a 4-dimensional Minkowski space [5]. Holographic duality prescribes the identification between the partition function of the conformal field theory and the one of the string theory on $A d S_{5}$ [6]. The correspondence determines a dictionary [6,7] according to which a local gaugeinvariant $p$-form with conformal dimension $\Delta$ is dual to a 5-dimensional field with mass $m_{5}$ given by $m_{5}^{2} R^{2}=(\Delta-p)(\Delta+p-4), R$ being the curvature radius of the anti-de Sitter space. A holographic description of QCD at finite temperature can be obtained by modifying the metric on the AdS space; I use this method for discussing the stability of the hybrid configurations against thermal fluctuations in comparison with other quark and gluon bound states.

\section{Mass spectrum at $T=0$}

In Poincaré coordinates the line element of the anti-de Sitter space can be written as

$$
d s^{2}=\frac{R^{2}}{z^{2}}\left(d t^{2}-d \vec{x}^{2}-d z^{2}\right) \quad z>0,
$$

where $(t, \vec{x})$ are the ordinary 4-dimensional coordinates, and $z$ is the fifth holographic coordinate. The boundary of the $A d S_{5}$ space is a compactification of Minkowski space [6] and corresponds to the condition $z=0$. This framework aims at reproducing QCD properties through a projection on the boundary of the gravity theory defined in the $A d S$ space (bulk). However, the AdS/CFT correspondence has been conjectured for a conformal gauge theory, while QCD is not conformal invariant. A bottom-up strategy for breaking this symmetry requires the introduction of an infrared scale in the holographic space. I adopt the soft-wall model, in which a background dilaton field $\phi(z)=c^{2} z^{2}$ involving a dimensionful scale $c=O\left(\Lambda_{Q C D}\right)$ implements a smooth cutoff of the holographic space $[8,13]$; in the so-called hard-wall model, the conformal symmetry is broken by restricting the coordinate $z$ to a maximum value [9]. The hybrid $1^{-+}$meson can be represented by the QCD local operator $J_{\mu}^{a}=\bar{q} T^{a} G_{\mu \nu}^{a} \gamma^{v} q$, with $G_{\mu \nu}^{a}$ the gluon field strength tensor and $T^{a}$ flavour matrices normalized to $\operatorname{Tr}\left[T^{a} T^{b}\right]=\delta^{a b} / 2$. The dual field is a 1 -form, $H_{\mu}=H_{\mu}^{a} T^{a}$, with mass $m_{5}^{2} R^{2}=8$ and dynamics described by a Proca-like action:

$$
S=\frac{1}{k} \int d^{5} x \sqrt{g} e^{-c^{2} z^{2}} \operatorname{Tr}\left[-\frac{1}{4} F^{M N} F_{M N}+\frac{1}{2} m_{5}^{2} H_{M} H^{M}\right],
$$

where $F_{M N}=\partial_{M} H_{N}-\partial_{N} H_{M}, M$ and $N$ 5-dimensional indices, $g$ the determinant of the metric (1) and $k$ a parameter making the action dimensionless. The mass spectrum and normalised eigenfunctions of the $1^{-+}$hybrid states

$$
\begin{aligned}
q_{n}^{2} & =M_{n}^{2}=4 c^{2}(n+2), \\
H_{n} & =\sqrt{\frac{2 n !}{(n+3) !}} c^{4} z^{4} L_{n}^{3}\left(c^{2} z^{2}\right),
\end{aligned}
$$

with $n=0,1,2, \ldots$ and $L_{n}^{m}$ the generalized Laguerre polynomial, can be obtained from the EulerLagrange equation for the transverse component of the bulk field $H_{\perp}^{\mu}\left(\partial_{\mu} H_{\perp}^{\mu}=0\right)$ [4].

Another analysis of the $1^{-+}$meson properties can be performed by means of the two-point correlation function of the local operator $J_{\mu}^{a}$

$$
\Pi_{\mu \nu}^{a b}=i \int d^{4} x e^{i q x}\left\langle 0\left|T\left[J_{\mu}^{a}(x) J_{\nu}^{b}(0)\right]\right| 0\right\rangle=-\left(\eta_{\mu \nu}-\frac{q_{\mu} q_{v}}{q^{2}}\right) \frac{\delta^{a b}}{2} \Pi^{\perp}\left(q^{2}\right)+\frac{q_{\mu} q_{v}}{q^{2}} \frac{\delta^{a b}}{2} \Pi^{\|}\left(q^{2}\right),
$$




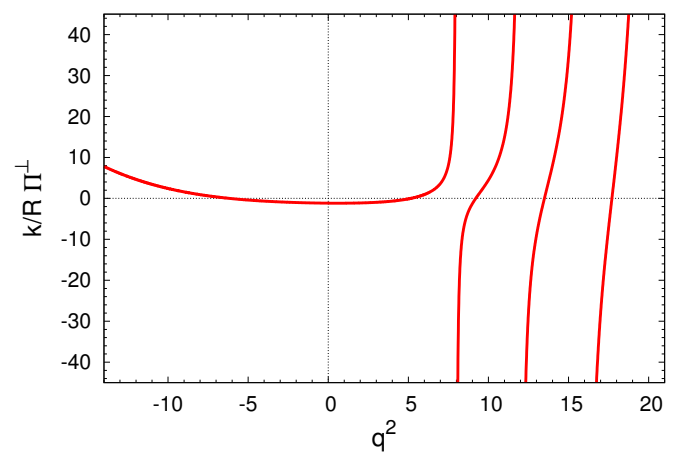

Figure 1. Regularized $\Pi^{\perp}\left(q^{2}\right)$ transverse invariant function; $c$ is set to one.

where the decomposition in a transverse and a longitudinal contribution has been used. Holographic QCD employs the weak version of the AdS/CFT duality [10], relating the strongly-coupled theory on the boundary to the supergravity theory in the bulk. In this limit the identification between the dual partition functions can be written as

$$
\Pi_{\mu \nu}^{a b}\left(q^{2}\right)=\left.\frac{\delta^{2} S_{o s}}{\delta H_{0}^{a \mu} \delta H_{0}^{b v}}\right|_{H_{0}=0}
$$

where $S_{o s}$ is the on-shell supergravity action (2) and $H_{0}^{\mu}\left(q^{2}\right)$ is the source, related to the hybrid field $H^{\mu}(z, q)=H\left(z, q^{2}\right) H_{0}^{\mu}\left(q^{2}\right)$ by the bulk-to-boundary propagator $H\left(z, q^{2}\right)$. A comparison between the expressions (5) and (6) allows one to determine the transverse function $\Pi^{\perp}\left(q^{2}\right)$, whose poles coincide with the mass spectrum (3), as shown in fig. 1 [4]. The residues can be calculated as well.

The soft-wall mass spectra of the various QCD mesonic bound states comprising quarks and gluons (Table 1) are Regge trajectories $M_{n}^{2} \simeq n$, having all the same slope. The mass of the lowest-lying hybrid state is $M_{0} \sim 1.1-1.3 \mathrm{GeV}$, depending on the way the parameter $c$ is fixed from the $1^{--}$spectrum $[8,13]$. The mass is lower than the predictions obtained by other approaches [2], and suggests $\pi_{1}(1400)$ as the lightest $1^{-+}$meson. Moreover, the mass spectrum (3) gives an intriguing description of the radial excited $1^{-+}$states, as it can be deduced comparing the prediction $M_{1} / M_{0}=\sqrt{3 / 2} \sim 1.22$ with the experimental ratio $M\left(\pi_{1}(1600)\right) / M\left(\pi_{1}(1400)\right) \sim 1.22$. According to this observation, one can interpret $\pi_{1}(1400)$ and $\pi_{1}(1600)$ as the lowest-lying and the first-excited state of the $1^{-+}$spectrum, respectively. In the hard-wall model, where the spectrum behaves as $M_{n}^{2} \simeq n^{2}$, the mass of the lowest-lying state is closer to $M\left(\pi_{1}(1400)\right)$ than the soft-wall prediction, but the first radial excitation is heavier [14].

Table 1. Mass spectrum of $q \bar{q}$, glueballs and hybrids predicted in the soft-wall model.

\begin{tabular}{lllll}
\hline$J^{P C}$ & $1^{--}(q \bar{q})[8]$ & $0^{++}(q \bar{q})[11]$ & $0^{++}$(glueball) [12] & $1^{-+}[4]$ \\
\hline$M_{n}^{2}$ & $c^{2}(4 n+4)$ & $c^{2}(4 n+6)$ & $c^{2}(4 n+8)$ & $c^{2}(4 n+8)$ \\
\hline
\end{tabular}




\section{Stability against thermal effects}

The spectral function $\rho\left(q^{2}\right)$ of $\Pi^{\perp}$ in (5), represented at zero temperature by an infinite number of delta functions centered at the eigenvalues of the mass spectrum, can be evaluated at increasing temperature in order to examine in-medium effects on hadron properties.

A possible framework for performing this analysis employs the AdS-Black Hole metric

$$
d s^{2}=\frac{R^{2}}{z^{2}}\left[\left(1-z^{4} / z_{h}^{4}\right) d t^{2}-d \vec{x}^{2}-\frac{d z^{2}}{1-z^{4} / z_{h}^{4}}\right] \quad 0<z<z_{h},
$$

with the position of its horizon $z_{h}$ depending on the temperature, $z_{h}=1 /(\pi T)$. From the action (2) and the geometry (7), the bulk-to-boundary propagator $H^{\perp}\left(z, q^{2}\right)$ can be computed in the meson restframe, $\vec{q}=0$. The resulting spectral function is shown in fig. 2 for a few values of the temperature $t=T / c$ [4]. The broadening of the peaks and their shift towards lower values of the squared mass when the temperature is increased, is visible. A melting temperature, indicating the dissociation of the hybrid states, can be identified from fitting the lowest-lying peak of the spectral function with a Breit-Wigner formula [4]. Table 2 collects the computed melting temperatures of various hadronic states. Thermal dissociation can also be described by means of a quark-gluon binding potential, which gets a shape preventing the formation of hybrid bound states at a critical temperature similar to the one inferred from the spectral function [4]. The analyses show that hybrid mesons melt at a lower temperature compared to light vector and scalar mesons, and scalar glueballs. In energy units, the hybrid $1^{-+}$melting temperature $T_{\text {melting }}$ is quite low, and depends on the way $c$ has been fixed, ranging approximately from 40 to $50 \mathrm{MeV}$.

A deconfinement phase transition can be introduced in order to describe the thermal response of the spatial string tension, which is temperature independent below $\sim 210 \mathrm{MeV}$ and rapidly increasing above this critical value [17]. Another way of performing the analysis of deconfinement includes the Hawking-Page transition between a low-temperature confined phase described by Thermal AdS metric and a high-temperature deconfined phase described by $A d S$ metric with black hole $[18,19]$.

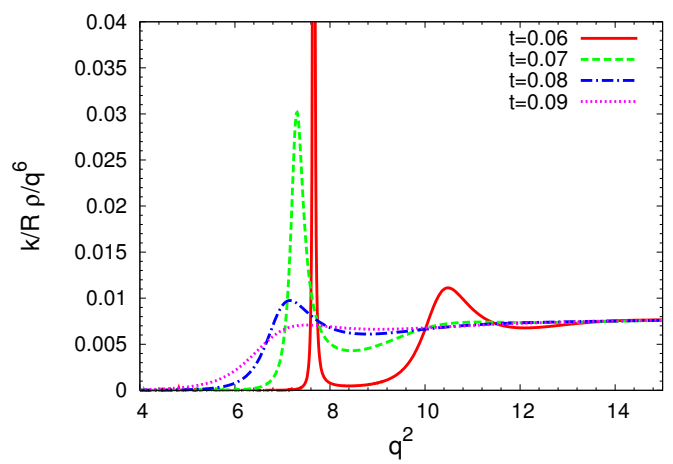

Figure 2. Spectral function of $1^{-+}$mesons at different values of temperature, with $c=1$.

Table 2. Melting temperature of $q \bar{q}$, glueball and hybrid states predicted in the soft-wall model.

\begin{tabular}{lllll}
\hline$J^{P C}$ & $1^{--}(q \bar{q})[15]$ & $0^{++}(q \bar{q})[16]$ & $0^{++}$(glueball) [16] & $1^{-+}[4]$ \\
\hline$t=T / c$ & 0.23 & 0.18 & 0.12 & 0.10 \\
\hline
\end{tabular}


The transition occurs at the temperature $T_{H P}(\sim 190 \mathrm{MeV}$ in the soft-wall model) for which the difference between the free energies of the two geometries changes sign [20]. Since the confinedphase spectral function equals the zero-temperature one, and $T_{H P}$ is considerably higher than $T_{\text {melting }}$, the Hawking-Page scheme describes thermal dissociation as a discontinuous process. Hadronic states retain their zero-temperature spectrum despite thermal fluctuations in the confined phase and suddenly disappear from the spectrum (i.e. they melt) at deconfinement.

\section{Conclusions}

In the soft-wall model the hybrid spectrum seems to reproduce the measured mass of the $1^{-+}$candidates. Hybrids are found to suffer of larger thermal instabilities with respect to other hadrons, and this could explain the difficulties in their detection. Further efforts are needed to develop a more refined dual model of finite temperature QCD, in which the nontrivial AdS-Black Hole picture of thermal dissociation holds at the more reasonable Hawking-Page temperature scale.

\section{Acknowledgements}

I thank P. Colangelo and F. Giannuzzi for collaboration, and F. Brünner for fruitful discussions.

\section{References}

[1] T. Barnes, Acta Phys. Polon. B 31, 2545 (2000)

[2] B. Ketzer, PoS QNP 2012, 025 (2012)

[3] J. Beringer et al., Phys. Rev. D 86, 010001 (2012)

[4] L. Bellantuono, P. Colangelo, F. Giannuzzi, Eur. Phys. J. C 74, 2830 (2014)

[5] J.M. Maldacena, Adv. Theor. Math. Phys. 2, 231 (1998)

[6] E. Witten, Adv. Theor. Math. Phys. 2, 253 (1998)

[7] S.S. Gubser, I.R. Klebanov, A.M. Polyakov, Phys. Lett. B 428, 105 (1998)

[8] A. Karch, E. Katz, D.T. Son, M.A. Stephanov, Phys. Rev. D 74, 015005 (2006)

[9] J. Erlich, E. Katz, D.T. Son, M.A. Stephanov, Phys. Rev. Lett. 95, 261602 (2005)

[10] J.D. Edelstein, J.P. Shock, D. Zoakos, AIP Conf. Proc. 1116, 265 (2009)

[11] P. Colangelo, F. De Fazio, F. Giannuzzi, F. Jugeau, S. Nicotri, Phys. Rev. D 78, 055009 (2008)

[12] P. Colangelo, F. De Fazio, F. Jugeau, S. Nicotri, Phys. Lett. B 652, 73 (2007)

[13] O. Andreev, V.I. Zakharov, Phys. Rev. D 74, 025023 (2006)

[14] H.-C. Kim, Y. Kim, JHEP 0901, 034 (2009)

[15] L.A.H. Mamani, A.S. Miranda, H. Boschi-Filho, N.R.F. Braga, JHEP 1403, 058 (2014)

[16] P. Colangelo, F. Giannuzzi, S. Nicotri, Phys. Rev. D 80, 094019 (2009)

[17] O. Andreev, V.I. Zakharov, Phys. Lett. B 645, 437 (2007)

[18] S.W. Hawking, D.N. Page, Commun. Math. Phys. 87, 577 (1983)

[19] E. Witten, Adv. Theor. Math. Phys. 2, 505 (1998)

[20] C.P. Herzog, Phys. Rev. Lett. 98, 091601 (2007) 
